\title{
Charge separation technique for metal-oxide-silicon capacitors in the presence of hydrogen deactivated dopants
}

\author{
Steven C. Witczak a) and Peter S. Winokur
Sandia. National Laboratories, P.O. Box 5800, MS-1083, Albuquerque, New Mexico 87185-1083 E CEIVED
Ronald C. Lacoe and Donald C. Mayer \\ Ronald C. Lacoe and Donald C. Mayer \\ The Aerospace Corporation, P.O. Box 92957, M2-244, Los Angeles, California 90009-2957 \\ (Received xx January 2000; accepted for publication xx February 2000) \\ An improved charge separation technique for metal-oxide-silicon (MOS) capacitors is presented \\ which accounts for the deactivation of substrate dopants by hydrogen at elevated irradiation \\ temperatures or small irradiation biases. Using high-frequency capacitance-voltage (C-V) \\ measurements, radiation-induced inversion voltage shifts are separated into components due to \\ oxide trapped charge, interface traps and deactivated dopants, where the latter is computed from a \\ reduction in Si capacitance. In the limit of no radiation-induced dopant deactivation, this approach \\ reduces to the standard midgap charge separation technique used widely for the analysis of room- \\ temperature irradiations. The technique is demonstrated on a p-type MOS capacitor irradiated with \\ ${ }^{60} \mathrm{Co} \gamma$-rays at $100{ }^{\circ} \mathrm{C}$ and zero bias, where the dopant deactivation is significant.
}

Midgap charge separation is a technique widely used to distinguish the contributions of oxide trapped charge and interface traps to radiation-induced threshold (or inversion) voltage shifts in metal-oxide-silicon (MOS) devices. ${ }^{1}$ The technique owes its popularity to uncomplicated analysis and simple implementation, requiring only high-frequency capacitance-voltage $(\mathrm{C}-\mathrm{V})$ measurements for capacitor structures or current-voltage measurements for transistor structures. Assuming charge neutrality of the interface traps when the Fermi energy is at midgap, ${ }^{2}$ radiation-induced shifts in the midgap voltage are attributed to oxide trapped charge, while stretchout of the inversion and midgap voltages is attributed to interface traps. Among other simplifications, the technique assumes that the concentration of electrically active substrate dopants is constant with total dose.

Due to the complexing of hydrogen with substrate dopants, this assumption of constant doping concentration is often violated for irradiations of p-bulk devices performed under low electric fields or at elevated temperatures. ${ }^{3} \mathrm{Hy}-$ drogen released in the oxide during irradiation is known to neutralize many types of shallow acceptors (especially B) in Si primarily through two mechanisms. ${ }^{4-13}$ In the case of acceptor passivation, ${ }^{4-8} \mathrm{H}^{0}$ can deactivate $\mathrm{B}^{-}$by occupying a bond-centered position along a $<111>$ axis between a substitutional B site and a neighboring Si atom. In the case of acceptor compensation, ${ }^{9-13}$ electron-hole pair recombination occurs when $\mathrm{H}^{0}$ donates an electron to the $\mathrm{Si}$ conduction band and ionically bonds to $\mathrm{B}^{-}$. For either mechanism, the result is a reduction in the net concentration of electrically active dopants and a decrease in the semiconductor capacitance. In this letter, we present an improved charge separation technique for MOS capacitors, which accounts for the deactivation of substrate dopants by radiation-released hydrogen.

To illustrate its utility, the technique is demonstrated on a test capacitor from a radiation-hardened bipolar complementary metal-oxide-silicon process developed by Analog

\footnotetext{
a) Electronic mail: scwitcz@sandia.gov
}

Devices. The capacitor is fabricated with a $55 \mathrm{~nm}$ wet thermal oxide and a B-doped Si substrate having a pre-irradiation surface doping concentration of $8 \times 10^{17} \mathrm{~cm}^{-3}$. The capacitor was irradiated under zero bias with ${ }^{60} \mathrm{Co} \gamma$-rays at 10 $\operatorname{rad}\left(\mathrm{SiO}_{2}\right) / \mathrm{s}$ and $100{ }^{\circ} \mathrm{C}$. Prior to irradiation and following each of four $25 \mathrm{krad}\left(\mathrm{SiO}_{2}\right)$ exposures, high-frequency (1 $\mathrm{MHz}) \mathrm{C}-\mathrm{V}$ measurements were performed at room temperature by sweeping the gate bias in equilibrium from inversion to accumulation. To minimize the dissociation of hydrogen-acceptor pairs by minority carriers, ${ }^{8,10}$ the irradiations and measurements were performed in the dark.

The C-V characteristics are shown in Fig. 1, where the measured capacitances are normalized by the capacitance of the oxide. Following irradiation, the $\mathrm{C}-\mathrm{V}$ characteristics undergo a parallel shift due to net positive oxide trapped charge and stretchout due to interface traps. In addition, the capacitance measured in depletion and inversion decreases commensurately with dose. This reduction in capacitance is a direct indication of the neutralization of substrate accep-

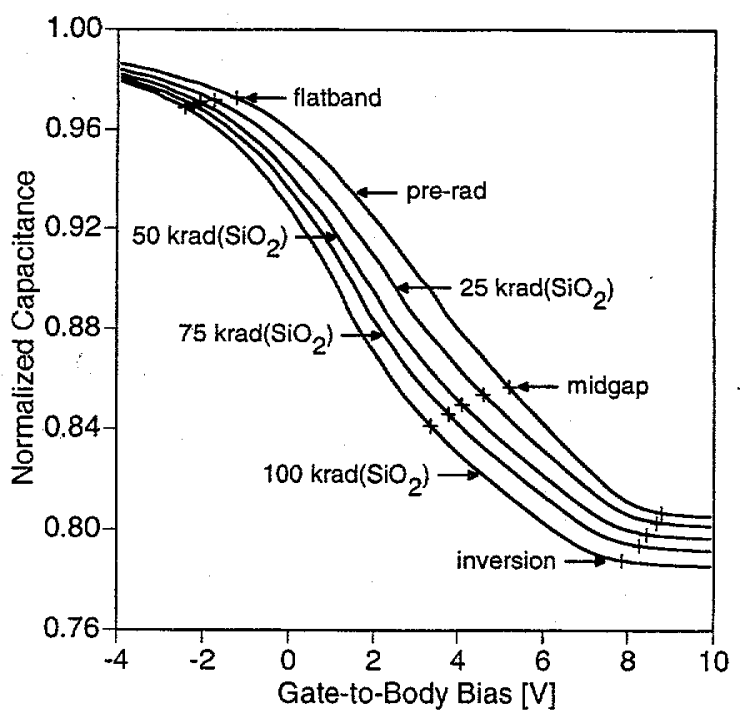

FIG. 1. Effect of total dose on the C-V characteristics for unbiased irradiation at $100{ }^{\circ} \mathrm{C}$. The capacitance decrease in depletion and inversion is a direct indication of the neutralization of $\mathrm{Si}$ acceptors by hydrogen. 


\section{DISCLAIMER}

This report was prepared as an account of work sponsored by an agency of the United States Government. Neither the United States Government nor any agency thereof, nor any of their employees, make any warranty, express or implied, or assumes any legal liability or responsibility for the accuracy, completeness, or usefulness of any information, apparatus, product, or process disclosed, or represents that its use would not infringe privately owned rights. Reference herein to any specific commercial product, process, or service by trade name, trademark, manufacturer, or otherwise does not necessarily constitute or imply its endorsement, recommendation, or favoring by the United States Government or any agency thereof. The views and opinions of authors expressed herein do not necessarily state or reflect those of the United States Government or any agency thereof. 


\section{DISCLAIMER}

\section{Portions of this document may be illegible in electronic image products. Images are produced from the best available original document.}


tors by hydrogen. ${ }^{4-13}$ Flatband, midgap and inversion voltages are indicated in the figure for clarity. Because the Si surface potential is a function of the concentration of electrically active dopants, the dopant neutralization leads directly to shifts in these voltages. The principal advantage of this technique over the existing one is that it accounts for the effect of doping changes on the electrostatic potential. Once dopant-related shifts in the inversion and midgap voltages are resolved, inversion voltage shifts due to oxide trapped charge and interface traps can be determined in the usual way by assuming charge neutrality of the interface traps when the Fermi energy is at midgap. ${ }^{2}$

The midgap and inversion voltages are found from the $\mathrm{C}-\mathrm{V}$ characteristics as the gate biases corresponding to the midgap and inversion capacitances. The high-frequency capacitances at midgap and inversion are found by evaluating $^{14}$

$$
C\left(\phi_{s}\right)=\frac{C_{o x} C_{S i}\left(\phi_{s}\right)}{C_{o x}+C_{S i}\left(\phi_{s}\right)}
$$

at the surface potentials $\phi_{s}=\phi_{f}$ and $\phi_{s}=2 \phi_{f}$, respectively, where, for uniform doping, the Si capacitance per unit area is approximated by

$$
C_{S i}\left(\phi_{s}\right)=\left[\frac{q \varepsilon_{S i} \varepsilon_{0} N_{B}}{2\left(\phi_{s}-k T / q\right)}\right]^{\frac{1}{2}},
$$

and the Fermi potential is defined as

$$
\phi_{f}=\frac{k T}{q} \ln \left(\frac{N_{B}}{n_{i}}\right) .
$$

In these expressions, $C_{o x}$ is the oxide capacitance per unit area, $k T$ is the thermal energy, $q$ is the electronic charge, $\varepsilon_{S i} \varepsilon_{0}$ is the permittivity of $\mathrm{Si}, n_{i}$ is the intrinsic carrier concentration of $\mathrm{Si}$, and $N_{B}$ is the net concentration of electrically active dopants.

The pre- and post-irradiation net doping concentrations are obtained by iteratively solving ${ }^{15}$

$$
\frac{C_{o x}}{C_{\min }}=1+C_{o x}\left[\frac{4 k T}{\varepsilon_{S i} \varepsilon_{0} q^{2} N_{B}} \ln \left(\frac{N_{B}}{n_{i}}\right)\right]^{\frac{1}{2}},
$$

where $C_{\min }$ is the minimum high-frequency capacitance per unit area. For nonuniform doping profiles, improved estimates of the midgap and inversion capacitances can be obtained using numerical simulation tools, where the doping profiles are extracted in the presence of interface traps using low- and high-frequency $\mathrm{C}-\mathrm{V}$ measurements. ${ }^{16,17}$

Assuming charge neutrality of the interface traps when the Fermi energy is at midgap, ${ }^{2}$ inversion voltage shifts due to radiation-induced net positive oxide trapped charge and interface traps, respectively, are estimated from

$$
\Delta V_{o t}=\left(V_{m g}-V_{m g}^{o}\right)_{p o s t}-\left(V_{m g}-V_{m g}^{o}\right)_{p r e},
$$

and

$$
\Delta V_{i t}=\left(V_{s o}-V_{s o}^{0}\right)_{p o s t}-\left(V_{s o}-V_{s o}^{0}\right)_{p r e},
$$

where $V_{m g}$ is the midgap voltage, $V_{s o}=V_{i n \nu}-V_{m g}$ is defined as the stretchout between the inversion and midgap voltages, the subscripts pre and post refer to the measurement time relative to radiation exposure, and the superscript 0 denotes an ideal value assuming the absence of oxide defects. Equation (5) states that the inversion voltage shift due to oxide trapped charge equals the portion of the midgap voltage shift not due to dopant neutralization, where the two ideal terms account for the change in net doping concentration. Similarly, (6) expresses the inversion voltage shift due to interface traps as the part of the stretchout voltage shift not due to dopant neutralization, where the net doping change is reflected in two more ideal terms.

Theoretical values for the midgap and inversion voltages, respectively, are evaluated at $V_{m g}{ }^{0}=V_{G}{ }^{0}\left(\phi_{s}=\phi_{f}\right)$ and $V_{i n y}{ }^{0}=V_{G}{ }^{0}\left(\phi_{s}=2 \phi_{f}\right)$, where ${ }^{18}$

$$
V_{G}^{O}\left(\phi_{s}\right)=\phi_{s}+\frac{1}{C_{o x}}\left(2 q \varepsilon_{S i} \varepsilon_{0} N_{B} \phi_{s}\right)^{\frac{1}{2}}
$$

describes the ideal relationship between the gate voltage and the surface potential. The fact that this relationship excludes nonidealities such as oxide trapped charge and interface traps is irrelevant to accounting for the dopant neutralization, since we are interested in shifts in $V_{m g}$ and $V_{i n v}$ due to net doping changes and not their absolute values. ${ }^{\text {b) }}$

An additional component of inversion voltage shift due to dopant deactivation is computed directly from the change in net doping concentration using

$$
\Delta V_{d o p}=\left(V_{i n v}^{O}\right)_{p o s t}-\left(V_{i n v}^{o}\right)_{p r e} .
$$

Since this component is obtained independently of the oxide defects, the radiation-induced inversion voltage shift can be expressed as

$$
\Delta V_{i n v}=\Delta V_{o t}+\Delta V_{i t}+\Delta V_{d o p} .
$$

In the limit of no radiation-induced dopant neutralization, this approach reduces to the standard midgap charge separation technique, ${ }^{1}$ where the pre- and post-irradiation ideal terms in (5), (6) and (8) cancel to yield the components $\Delta V_{o t}=\Delta V_{m g}, \Delta V_{i z}=\Delta V_{s o}$, and $\Delta V_{d o p}=0$.

Fig. 2 shows the charge-separated components of inver-

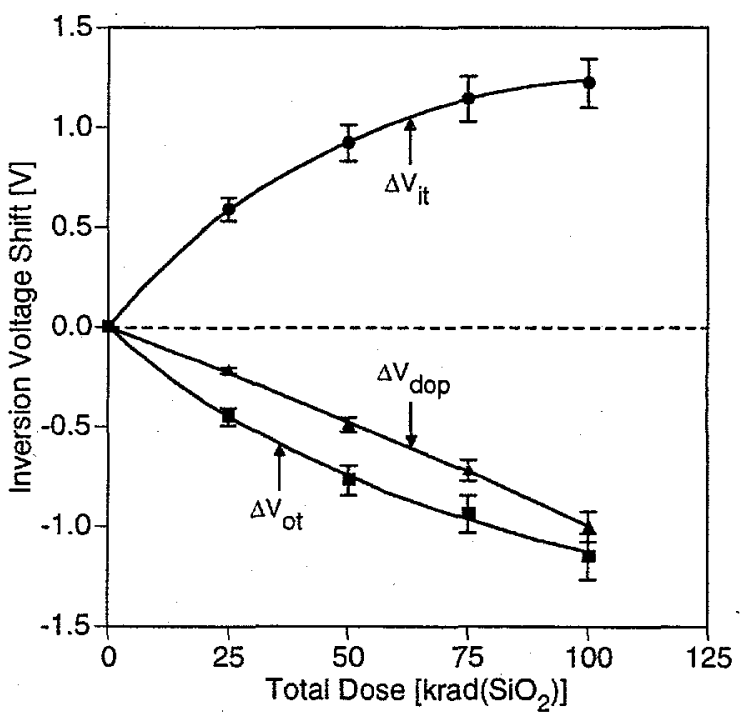

FIG. 2. Effect of total dose on the charge-separated inversion voltage shifts obtained from $\mathrm{C}-\mathrm{V}$ characteristics of the previous figure. Hydrogen neutralization of the $\mathrm{Si}$ acceptors shifts the inversion voltage negative.

b) The gate-to-body work function difference, $\phi_{g} b$, is neglected in this analysis. Over the range of doses examined, radiation-induced changes in $\phi_{g b}$ are less than $1 \mathrm{mV}$ for these capacitors. 
sion voltage shift as a function of total dose. Radiationinduced interface traps shift the inversion voltage positive, while net positive oxide trapped charge and dopant neutralization shift the inversion voltage negative. The relative contributions of the components emphasize the importance of accounting for dopant neutralization when distinguishing radiation-induced defects. Over the range of doses examined, $\Delta V_{\text {dop }}$ approaches $\Delta V_{o t}$ and $\Delta V_{i t}$ in magnitude. The largest error in the components results from resolving radiation-induced shifts in $V_{m g}$ and $V_{s o}$ in the presence of nonuniform doping profiles. Previous work suggests that the neutralization of dopants occurs preferentially near the Si surface. ${ }^{9}$ For the capacitor studied, numerical simulations show that a $40 \%$ variation in doping concentration over the maximum depletion width leads to errors of less than $0.2 \mathrm{~V}$ for $V_{m g}$ and $V_{s o}$.

Densities of radiation-induced oxide trapped charge and interface traps, respectively, are plotted as a function of total dose in Figs. 3(a) and 3(b), where the densities are related to

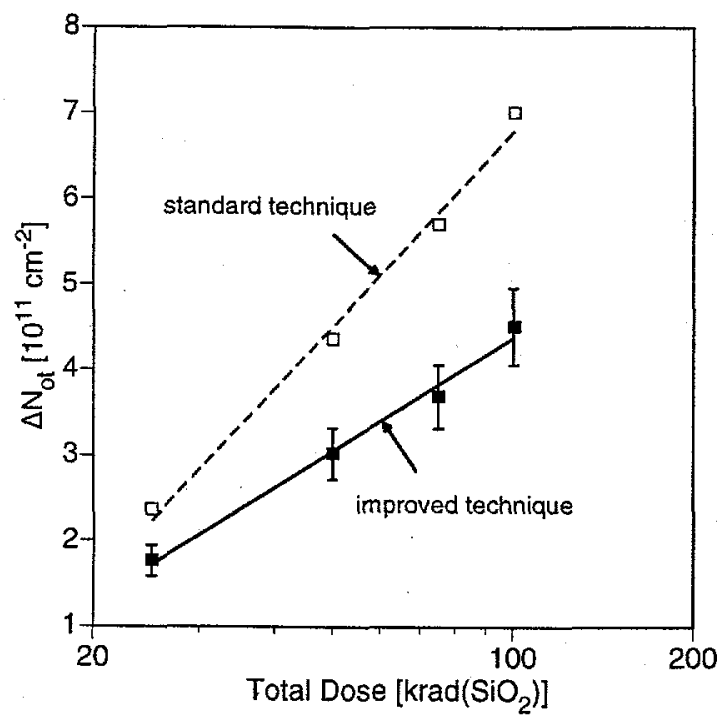

(a)

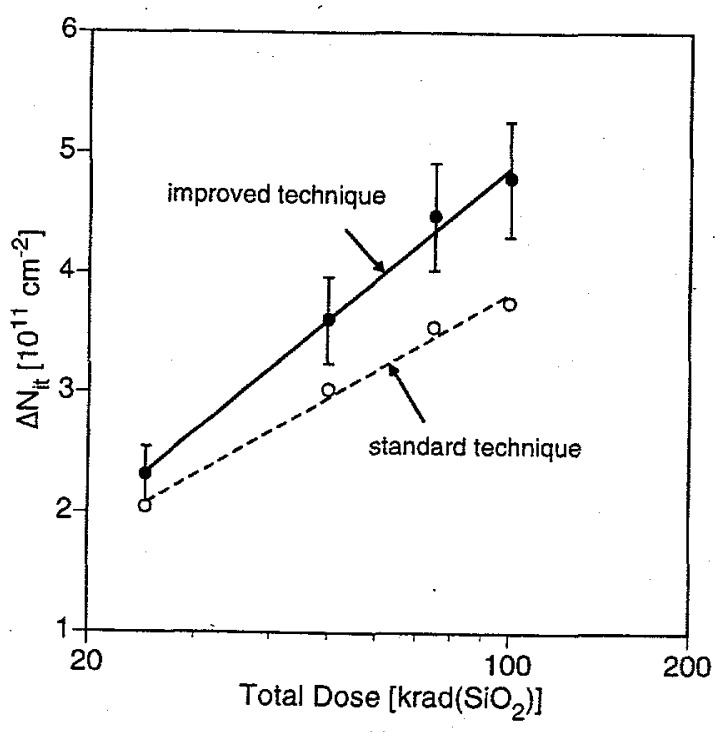

(b)

FIG. 3. Effect of total dose on the buildup of (a) net positive oxide trapped charge and (b) interface traps in the test capacitor. Neglecting the neutralization of dopants by hydrogen leads to an overestimation of net positive oxide trapped charge and an underestimation of interface traps. the appropriate inversion voltage shifts through the proportionality constant $C_{o x} / q .{ }^{1}$ For comparison, the densities are computed using both the improved and standard techniques. The standard technique overestimates the buildup of oxide trapped charge by as much as $60 \%$, because it erroneously attributes dopant-related shifts in the midgap voltage to oxide trapped charge. Similarly, the standard technique underestimates the buildup of interface traps, because it fails to account for the fact that the dopant deactivation shifts the inversion voltage more negative than it does the midgap voltage.

To summarize, we have presented an improved charge separation technique for MOS capacitors, which accounts for the deactivation of substrate dopants by hydrogen at elevated irradiation temperatures or small irradiation biases. The technique can be applied without regard to details of the hydrogen source, hydrogen release mechanisms or dopant deactivation mechanisms involved. Using high-frequency $\mathrm{C}-\mathrm{V}$. measurements, radiation-induced inversion voltage shifts are separated into components due to oxide trapped charge, interface traps and deactivated dopants, where the latter is computed from a reduction in Si capacitance. By accounting for the dopant neutralization, improved estimates of the densities of radiation-induced oxide trapped charge and interface traps are obtained. In the limit of no radiationinduced dopant deactivation, this approach reduces to the standard midgap charge separation technique ${ }^{1}$ used widely for the analysis of room-temperature irradiations.

The authors are indebted to M. DeLaus and A. Martinez of Analog Devices, Inc. for furnishing the test capacitors. Sandia is a multiprogram laboratory operated by Sandia Corporation, a Lockheed Martin Company, for the U.S. Department of Energy under Contract No. DE-AC0494AL85000. Work performed at the Aerospace Corporation was supported by the U.S. Air Force Space and Missile Center under Contract No. F04701-93-C-0094.

${ }^{1}$ P. J. McWhorter and P. S. Winokur, Appl. Phys. Lett. 48, 133 (1986).

${ }^{2}$ P. M. Lenahan and P. V. Dressendorfer, J. Appl. Phys. 55, 3495 (1984).

${ }^{3}$ S. C. Witczak, R. C. Lacoe, D. C. Mayer, D. M. Fleetwood, R. D. Schrimpf and K. F. Galloway, IEEE Trans. Nucl. Sci. 45, 2339 (1998).

${ }^{4}$ P. J. Pankove, R. O. Wance and J. E. Berkeyheiser, Appl. Phys. Lett. 45, $1100(1984)$.

${ }^{5}$ G. G. DeLeo and W. B. Fowler, Phys. Rev. B 31, 6861 (1985).

${ }^{6}$ M. Stavola, Appl. Phys. Lett. 50, 1086 (1987).

${ }^{7}$ C. P. Herrero and M. Stutzmann, Phys. Rev. B 38, 12668 (1988).

${ }^{8}$ N. M. Johnson, C. Doland, F. Ponce, J. Walker and G. Anderson, Physica B 170, 3 (1991).

${ }^{9}$ C.-T. Sah, J. Y.-C. Sun and J. J.-T. Tzou, Appl. Phys. Lett. 43, 204 (1983).

${ }^{10}$ A. J. Tavendale, D. Alexiev and A. A. Williams, Appl. Phys. Lett. 47, 316 (1985).

${ }^{11}$ Zundel, E. Courceile, A. Mesli, J. C. Muller and P. Siffert, Appl. Phys. A 40, 67 (1986).

${ }^{12}$ P. J. H. Denteneer, C. G. Van de Walle and S. T. Pantelides, Phys. Rev. B 39, 10809 (1989).

${ }^{13}$ S. J. Pearton, J. W. Corbett and J. T. Bernstein, Physica B 170, 85 (1991).

${ }^{14}$ E. H. Nicollian and J. R. Brews, MOS (Metal Oxide Semiconductor) Physics and Technology (Wiley, New York, 1982), Chap. 4.

${ }^{15}$ B. E. Deal, A. S. Grove, E. H. Snow and C. T. Sah, J. Electrochem. Soc. 112, 308 (1965).

${ }^{16}$ J. R. Brews, J. Appl. Phys. 44, 3228 (1973).

${ }^{17}$ Y. Zohta, Solid-State Electron. 17, 1299 (1974).

${ }^{18}$ R. F. Pierret, Field Effect Devices, Modular Series on Solid State Devices Vol. 4, 2nded. (Addison-Wesley, New York, 1990), Chap. 2. 